\title{
Retrospection on localization techniques for positioning nodes in Wireless Sensor Networks
}

\author{
Panimalar Kathiroli, Kanmani. S
}

\begin{abstract}
Wireless Sensor Networks have highly scattered, self-organized nodes that can detect, compute, and transmit the information collected at different nodes in the network. These nodes spread over a specific topographical zone. They enhance the instantaneous formation of the network. Knowing the current location of a node is a crucial and cardinal requirement for any application promulgated in WSN. Once the locations of the sensor nodes can be precisely positioned, there are ample of probabilities for the data transmission of the network to be excelling inefficiency. Location responsiveness enables essential network features such as coverage, routing, deployment, topology control, clustering, boundary discovery, target tracking, rescue, and other location services. Hence, WSN localization has become a breath and backbone arena that ostentatiously attracted significant research interest. Our work traces a compilation of all the dynamic research in sensor networks on localization techniques and emanates eminent understanding of it.
\end{abstract}

Keywords: Localization, Position, Sensor, Sensor Networks.

\section{INTRODUCTION}

A cluster of sensor nodes that are linked together in a sensor field to carry on an application-oriented task [4] is called Wireless Sensor Network (WSN). The nodes situated at various places in the sensor field. They have the potential to sense data in any environment, process the data and communicate them to the neighborhood sensor nodes. Amid these, at least one node shall assist as a sink node that is proficient of cooperating with the user [24]. Either openly or implicitly with help of internodes, these nodes align as a link.

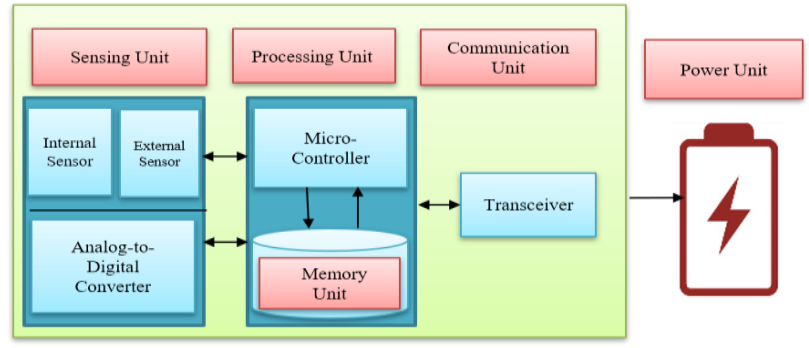

Fig.1. Components of Sensor node

The various components of the sensor node as exhibited in Figure 1 are sensing unit, processing unit, a communication unit, memory unit, and power unit [25]. The responsibility of a sensing unit is just the transfer of messages in short distances.

Revised Manuscript Received on February 05, 2020.

* Correspondence Author

Panimalar. K, Research*, Department of Computer Science and Engineering, Pondicherry Engineering College, Puducherry, India.

Dr. S. Kanmani, Faculty, Department of Computer Science and Engineering, Pondicherry Engineering College, Puducherry, India

(c) The Authors. Published by Blue Eyes Intelligence Engineering and Sciences Publication (BEIESP). This is an open access article under the CC BY-NC-ND license (http://creativecommons.org/licenses/by-nc-nd/4.0/)

They comprised of an external and internal sensor. This assists in perceiving the information from the physical world. To convert the collected analog signals to digital signals, they provided with an Analog-to-Digital Converter (ADC) [1]. The processing unit will process the received signals. Else, to the above cited it has the accountability of controlling other components in the node that enables to process, run algorithms and cooperate with other nodes. Memory unit has an inbuilt memory unit like Random Access Memory (RAM), Read-Only Memory (ROM), or external storage [26]. The communication unit's job is to commune with other nodes and the rest of the segments of WSN. The transceiver is the most power-consuming unit. The power unit is responsible for supplying node energy. Small batteries or capacitors are equipped in the sensor nodes to produce small-sized and low-cost devices [2].

\section{PERFORMANCE ISSUES IN WSN}

Unpredictable condition prevails in network's performance in the transmission of information. They are likely to cause hazards in the environment in the wireless networks.

\section{A. Hardware}

The sensor may be built-in or made of additional hardware. That results in a cost hike, more power consumption, and an increase in the size of the node. Further, the additional hardware needs elaborately considered with careful analysis for cost and low-power requisite [31].

\section{B. Time Synchronization}

The target is to proffer a period for local clocks of nodes in sensor networks. A crucial role is played frequently in real-time for this type of real-world monitoring applications. However, offering it becomes a perplexing task by its different challenging characteristics traits of sensor networks.

\section{Node Deployment}

The sharing of nodes relies on the application. Categorized as deterministic and randomized. During the random node distribution option of optimal clustering is essential to permit connectivity and enhance efficient energy network operation. Every sensor node should be self-managing and adapt to failure in any environment.

\section{Middleware}

This is a runtime environment bestows the hardware, operating systems, network, and applications. Middleware can synchronize multiple applications with system services and so it covers the complexity of the network.

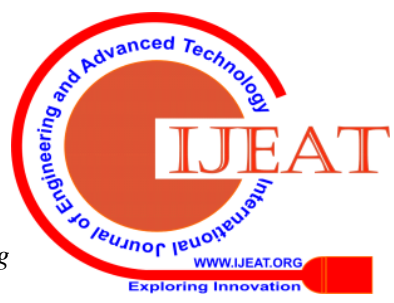




\section{E. Data Aggregation}

To collect data out of sensors and frame meaningful information after excluding the duplication. Innumerable protocols it is adapted to attain data transfer optimization and efficiency of energy. The minimal shortest route between the pairs of end nodes traced out by traditional address-centric routing. The data fusion-centric routing establishes routes leading the most extensive degree of data aggregation.

\section{F. Medium access schemes (MAC)}

MAC protocols operate the radio of the nodes that must possess low latency and high throughput directly in the network. For the variation in the topology, the network should adjust to the variations in the size and node density. Few nodes may join, some may perish over time, and the meagre figure of nodes may migrate to separate sites.

\section{G. Quality of Service (QoS)}

Energy management is essential than the class of data sent. The network brings down characteristics of the results while the energy gets exhausted or drained. Energy-aware routing protocols needed for lessening the energy dissipation in the nodes. It aids in increasing the network's lifetime.

\section{H. Security}

WSN is susceptible to various threats and risks. A hacker may intrude into the integrity of the data, inject false messages, squander the resources of the network and convert the sensor node to adhere to them. For safety sake, security protocols should be used to address security issues.

\section{Network layer Issues}

The network layer liable aimed at issues in routing and in transfer of the data from nodes to the BS. It plays a vital role in divulging the power-efficient route to attain the network lifetime optimized. In route maintenance, the routing protocol used in case of failure of the node due to environmental interference. The routing protocol should allow redundancy because the nodes are data centric. This improves power and bandwidth utilization. Multi-path protocols used in case of a path failure.

\section{J. Operating System (OS)}

For efficient performance to deal with various entities such as memory and resources in a constrained environment, an OS framework is to be well equipped. The OS have inherent to minimize the depletion of energy. The choice can be application-specific and hardware independent [50].

\section{Transport layer Issues}

The transport layer provides end-to-end consistent message transfer that may suffer if the position of the nodes did not fix. The protocol should transmit the fragmented segments in order. It must be reliable for delivering data under extreme conditions. The limit in bandwidth results poor communication with loss of data.
In emerging WSN applications, it is crucial to know a global coordinate of the node to send and receive data. The network consists of known or beacon or reference or anchor nodes, which recognizes location. The nodes that do not know their locations called unknown or target node. The problem of establishing the location of the sensor's node after positioned is called localization [24]. Built on the data of the reference nodes, the locations can be determined using hop techniques. Firstly, the one-hop localization estimates the place of the unknown node by communicating directly with the reference node, i.e., a one-hop reference neighbor. Physical locations of these nodes obtained through the Global Positioning System or arrived according to the known distribution but not calculated assessed based on localization system. Even though the built-in GPS receiver used in finding the location that may be utilized point out the spot, nevertheless this is not adequate for WSNs since GPS receivers are not affordable and power consuming. The other technique is multi-hop localization. In vague to pinpoint the position of the unknown node through deploying the hop distance. The process of broadcasting the location coordinates by the multi-hop localization called flooding [62]. The steps in the localization process displayed in Figure 2 , here the input hangs on the estimation of distance or angle to the anchor node or using position based on the position. Then the localization algorithm is applied to get the output.

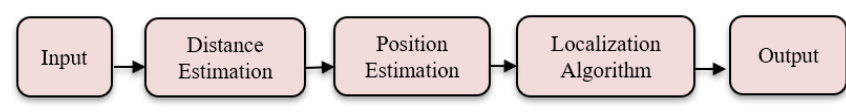

Fig.2. Steps in Localization

\section{CONTRIBUTION AND PAPER ORGANIZATION}

The remainder of the paper prepared as follows: Section IV discloses classification of localization algorithms in WSNs, followed by its assessment within the types. Section VII unfurls with comparative study on WSN Localization. Section VIII gives a brief retrospective discussion on results of state-of-the-art algorithm in localization. Finally, Section IX concludes, and future work is given.

\section{CLASSIFICATION OF LOCALIZATION ALGORITHM}

\section{Based on Computation or Data processing 1.1 Centralized localization algorithm}

The info from all the nodes sent to the BS, as shown in Figure 3(a). BS calculates the location and forward to respective neighbor. When sent through a discrete node computation cost and energy is low. The data transmission causes latency; hence, there is a lack of ability to access data in a proper way as well as insufficient scaling in the network.

\subsection{Distributed localization algorithm}

There is no clustering process as shown in Figure 3(b), and every node estimates its position. It brings down the bottleneck in traffic. Scalability and less storage requirements make the cost high [53].

\section{K. Localization}



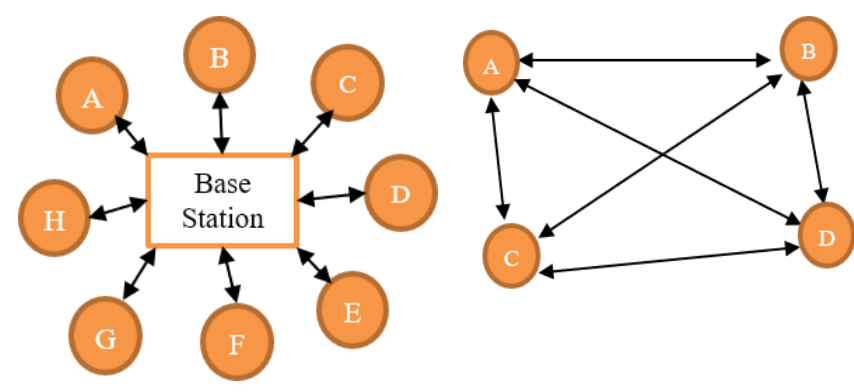

Fig.3(a). Centralized

Fig.3(b). Distributed

2. Based on Transmission range

2.1 Range -based localization algorithm

The techniques in localization consider the distance or angle [25] as the necessary parameters and define:

- Lateration: Distance between the nodes used as a parameter to evaluate the location.

- Angulation: Angle between the nodes used as a parameter to guess the location.

- Multilateration: The location of nodes estimated from more than three-node distances.

- Trilateration: The place of the node estimated from three-node distances. The target node location calculated by using the connection of three circles of the three nodes respectively. The coordinate of the unknown node listed as in Equation (1), (2), (3). Where A, B, C are nodes, $\left(X_{a}, Y_{a}\right),\left(X_{b}, Y_{b}\right),\left(X_{c}, Y_{c}\right)$ are coordinates of $A, B, C$ and $d_{a}$, $\mathrm{d}_{\mathrm{b}}, \mathrm{d}_{\mathrm{c}}$ are distances between the nodes

$$
\begin{aligned}
& \sqrt{\left(X-X_{a}\right)^{2}+\left(Y-Y_{a}\right)^{2}}=d_{a} \\
& \sqrt{\left(X-X_{b}\right)^{2}+\left(Y-Y_{b}\right)^{2}}=d_{b} \\
& \sqrt{\left(X-X_{c}\right)^{2}+\left(Y-Y_{c}\right)^{2}}=d_{c}
\end{aligned}
$$

- Triangulation: The position of the node

the angle of the target node from the known node. Trigonometric functions Sine and Cosine are used and represented in Equation (4), (5), (6), (7). Where A, B, C are angle of nodes, a, b, c are coordinates.

$$
\begin{aligned}
& \frac{a}{\sin A}=\frac{b}{\sin B}=\frac{c}{\sin C}=\text { constant } \\
& \operatorname{Cos} A=\frac{b^{2}+c^{2}-a^{2}}{2 b c} \\
& \operatorname{Cos} A=\frac{a^{2}+c^{2}-b^{2}}{2 a c} \\
& \operatorname{Cos} A=\frac{a^{2}+b^{2}-c^{2}}{2 a b}
\end{aligned}
$$

\section{(i)Timing-based Algorithm}

The distance amid two nodes estimated by measurement of a communication signal in terms of time.

\section{- Time of Arrival (TOA)}

It is the measurement of distance as given in Equation (8) between the beacon and target node as in Figure 4 and proportionate to the propagation time of the signal. It gives high accuracy and requires synchronization. Where $\mathrm{V}$ is velocity of signal, $\mathrm{t}_{1}, \mathrm{t}_{2}$ are signal sent and received time.

$$
\text { Distance }=v\left(t_{1}-t_{2}\right)
$$

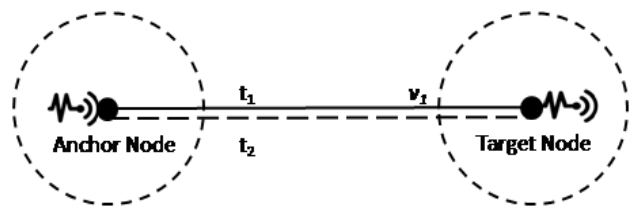

Fig.4. Time of Arrival

\section{- Time Difference of Arrival (TDOA)}

As stipulated in equation (9), TDOA be subject on the measurement of Time Of Arrival (TOA). This aids in measuring the signal of the transmitter to neighboring receiver nodes by the distance, as shown below in Figure 5. It has recorded high accuracy by its additional hardware. This results in excessive cost. Where $\mathrm{V}$ is velocity of signal and $t_{1}$, $t_{2}, t_{3}, t_{4}$, are signal sent and received time

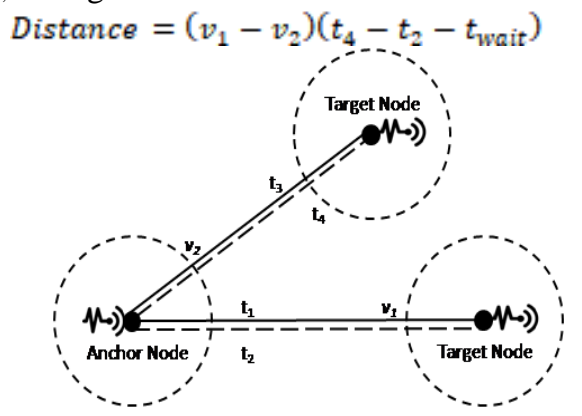

Fig. 5. Time Difference of Arrival

\section{(ii) Directionality-based Algorithm}

The direction of the signal received from a node is stately to guess the angle of the sender node.

\section{- Angle of Arrival (AOA)}

Each sensor evaluates the relative angles concerning the received radio signals. An antenna and complex hardware are required. Within a few degrees range of transmission, it gives high accuracy. It needs extensive signal processing that hikes the hardware cost.

(iii) Signal-strength-based Algorithm

Using the received signal, the distance between two nodes of theoretical or empirical models are computed

\section{- Received Signal Strength Indicator (RSSI)}

The widely practiced technique for indoor and outdoor environments especially for improving accuracy is RSSI. Also, it depends upon the radio frequency in the distance measurement between sender and receiver, as in Figure 6 . The resultant signal strength keeps diminishing, as distance to the receiver node is wider. The signal strength is inversely proportional to squared distance as explained in Equation (10). This indicator enormously related to obstacles that compel it hard for achieving a model mathematically in a real-world environment. Where $L$ is system loss factor, $P_{t}$ is transmitted power, $G_{r}$ is receiver antenna gain., $\lambda$ is wavelength of the signal, $G_{t}$ is transmitter antenna gain, $P_{r}(d)$ is Received power at a distance $\mathrm{d}$.

$$
P_{r}(d)=\frac{P_{t} G_{t} G_{r} \lambda^{2}}{(4 \pi)^{2} d^{2} L}
$$




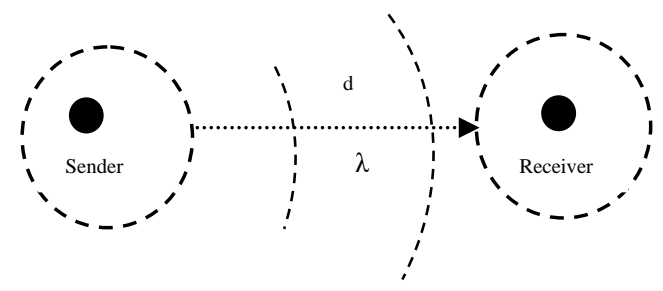

Fig. 6. Received Signal Strength Indicator (RSSI)

\subsection{Range-free localization algorithm}

It does not use distance to estimate the location instead uses detecting features like wireless connectivity, localization event detection and anchor node proximity. Due to the hardware limitations of these devices, the output is cost-effective.

\section{(i)Distance Vector Hop}

Through the hop count, DV hop detects the distance between nodes.

At least three anchor nodes are involved in broadcasting coordinates with hop count through the network [17]. Hop count gets incremented when a neighbor node receives data. Almost all known nodes compute the shortest route from other nodes, and all unknown nodes also compute the shortest route from all known nodes. Usually, unknown nodes use a triangulation routine to find their positions from three or more known nodes employing hop count to gauge the shortest distance [4]. The formula for average hop distance mentioned as the distance between two nodes in the number of hops. An example with five nodes A, B, C, D, and E shown in Figure 7 estimating its average hop length and distance between unknown to known node. Where a,b are anchors, $\mathrm{N}$ is Number of anchors in the network, Hopcount ${ }_{\mathrm{a}, \mathrm{b}}$ is the distance between anchor $\mathrm{A}$ to $\mathrm{B},\left(\mathrm{X}_{\mathrm{a}}, \mathrm{Y}_{\mathrm{a}}\right),\left(\mathrm{X}_{\mathrm{b}}, \mathrm{Y}_{\mathrm{b}}\right)$ are coordinates of anchors $\mathrm{A}$ and $\mathrm{B}, \mathrm{d}_{\mathrm{b}}$ is the distance between unknown to known node, AverageHopDistance $_{a}$ is hop Distance of unknown node, HopCount $_{b}$ is hop counter from unknown to anchor b.

\section{ALGORITHM}

Step 1: Receive broadcast packet from anchor node A, B Step 2: Initialize Hopcount $=0$

Step 3: Each node $N$ maintains: Table $\left(\left(\mathrm{X}_{\mathrm{a}}, \mathrm{Y}_{\mathrm{a}}\right),\left(\mathrm{X}_{\mathrm{b}}, \mathrm{Y}_{\mathrm{b}}\right)\right.$, Hopcount)

Step 4: if PacketReceived $\|$ || if Hopcount Rcvd $_{\text {Hopcount }}<$ Hopcos then Hopcount is updated, PacketReceived forwarded in the network , Hopcount++;

Step 5: if PacketReceived Hopcount $>$ Hopcount Then PacketReceived is discarded Until all the nodes know Minimum hopcount

Step 6: Calculate AverageHopDistance from unknown to known node

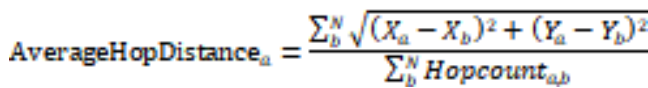

Step 7: Calculate the distance from unknown to known node

$$
\mathrm{d}_{\mathrm{b}}=\text { AverageHopDistance }_{\mathrm{a}} \mathrm{X} \text { HopCount }_{\mathrm{h}}
$$

Step 8: Calculate the unknown node Coordinates by Polygon method

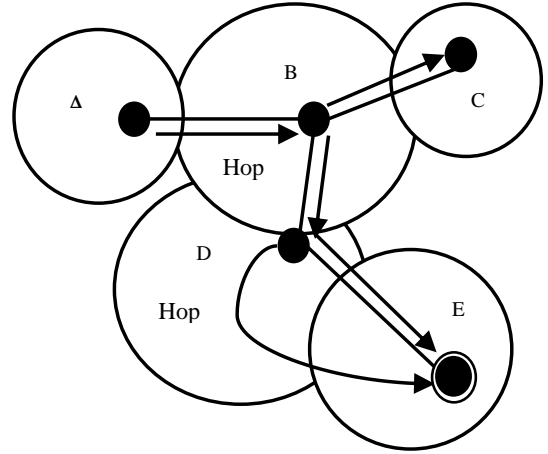

Fig.7. DV Hop Localization

\section{(ii) Hop Terrain}

Like the DV hop method, the hop terrain used in outcome of the distance concerning a known and an unknown node. There are two fragments in this method [54]. In the first fragment, an unknown node estimation its location from the known node by means of average hop distance formulation, which is the distance between nodes/total numbers of hops. Initial position evaluation as viewed in Figure 8; the second part is the transmission of the initial estimated position to the neighbor nodes. A node processes its position until the final position met using least square method [52].

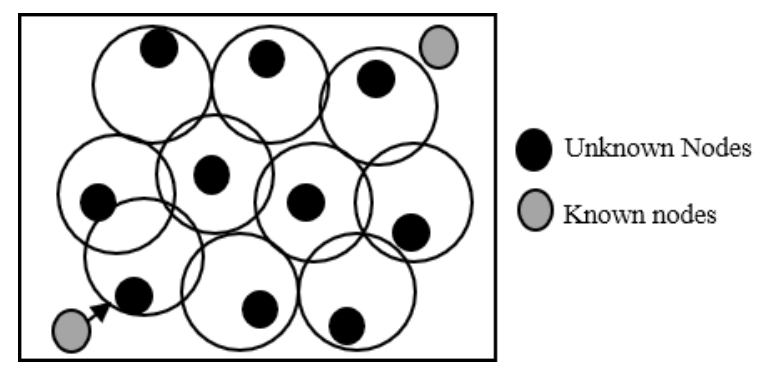

Fig.8. Hop terrain

\section{(iii) Approximate Point in Triangulation}

The known nodes avail position coordinates from external devices like GPS or transmitters in APIT. The unknown node gets place from overlapping triangles, as portrayed in Figure 9. Out of the known nodes, the unknown nodes retain a table with the information on known node ID, location, and signal strength after receiving signal messages. Unknown nodes choose on any three known nodes from the triangle area form known as the PIT (Point In Triangulation) test. The PIT test continues the precision of an unknown node positioned by arrangement of three known nodes.

\section{ALGORITHM}

Step 1: Receive location beacons $\left(\mathrm{X}_{\mathrm{i}}, \mathrm{Y}_{\mathrm{i}}\right)$ from $\mathrm{n}$ anchors. Step 2: Intersect $=\Phi / /$ the set of triangles in which i reside Step 3: For each triangle $T_{\mathbb{L}} \in\left(\begin{array}{l}n \\ 3\end{array}\right)$ triangles

Step 4: If (Point-In-Triangle-Test $\left.\left(\mathrm{T}_{\mathrm{i}}\right)==\mathrm{TRUE}\right)$

Step 5: Intersect $=$ Intersect $\cup\left(T_{0}\right]$

Step 6: If (accuracy (Intersect)>enough), Break

Step 7: Estimated Position=COG $\left(\cap T_{\mathbb{I}} \in\right.$ Intersect /*Center of gravity (COG) calculation*/ 


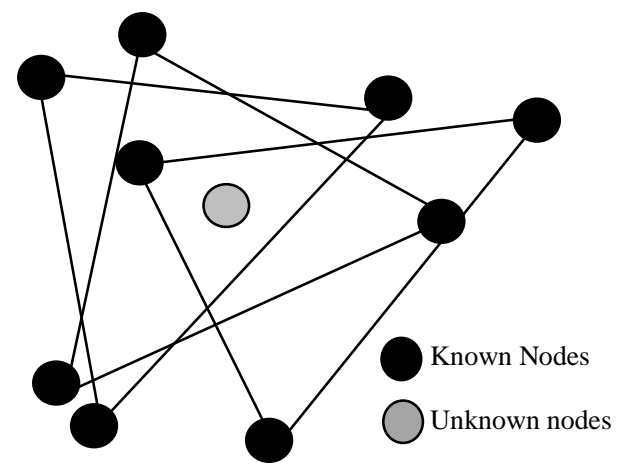

Fig.9. Approximate Point in Triangulation

\section{(iv) Gradient Algorithm}

Multilateration is deployed in the unknown node to sense its position. The gradient begins by known nodes and aids unknown nodes to find their location from three known nodes by opting multilateration. The initial value of hop count value is set to zero and increased while broadcasted to the neighboring nodes. Every sensor node pulls out the data on the shortest route from known nodes. The steps of the algorithm are initially the known node broadcasting signals that include coordinate and hop count value. Following this, the unknown node computes the shortest route amongst them and the known node through which it collects signals. Finally, an error equation used to get a minimum error in which node determines coordinate by multilateration.

\section{(v) Centroid Algorithm}

This algorithm demands a smaller number of computations with minimum communication costs. Among the algorithms, the centroid algorithm is the most straightforward range-free algorithm. Here all the unknown nodes find positions as the centroid of all acknowledged information from the known nodes within their respective range. However, each beacon node assumed to be inside a circle, this can interconnect with them. If there are four known nodes within a circular sort and there remains one unknown node, the estimated location for the beacons is the centroid value. By the usage of the centroid formula, the accuracy is high compared to other algorithms. However, the accuracy and the beacon node's density of the estimate location are subject to on the type of sharing; more uniform the network, more raises the localization accuracy.

\section{ALGORITHM}

Step 1: Receive the location from $n$ neighbor beacon nodes $\left(\mathrm{X}_{\mathrm{i}}, \mathrm{Y}_{\mathrm{i}}\right)$

Step 2: Then, Calculate location of unknown nodes $\left(\mathrm{X}, \mathrm{Y}^{\prime}\right)$ using centroid Formula

Step 3: if $n \geq 3$ then

$$
\begin{aligned}
& \mathrm{X} \text { - coordinate and } \mathrm{Y}-\text { coordinate calculated as } \\
& \left(\mathrm{X}_{v}, \mathrm{Y}^{\mathrm{v}}\right)=\frac{\sum_{\mathrm{i}=1}^{n} x_{\mathrm{i}}}{n}, \frac{\sum_{\mathrm{i}=1}^{n} Y_{\mathrm{i}}}{n} \quad \text { end if }
\end{aligned}
$$

Step 5: Repeated for all unknown nodes

\section{Based on Accuracy or granularity of information}

\subsection{Fine-grained localization algorithm}

Anchors are potential enough to know their locations by GPS or manual alignment [31].
The distance between estimated and neighbor node typically called reference points produce high accuracy information in location of the node. Trilateration or triangulation method is engaged to compute the location of the target node.

\subsection{Coarse-grained localization algorithm}

It estimates the target node location from its proximity to the available anchor nodes. To provide location of anchor node, place the node at stationary locations. Here location estimated by GPS or pre-setup from a location server.

\section{Based on Mobility \\ 4.1 Mobile localization algorithm}

The mobility of a node will give better performance in relations to cost, coverage, and accuracy. Challenges are inevitable when a mobile node in the network used to discover the path with minimum distance, rate of the localization and energy efficiency issues [36]. The difficulty of agility in WSNs has lately grown too much concern as uses of mobile sensor nodes increased [58].

\subsection{Static localization algorithm}

Some nodes are static and confined in one place. Many localization algorithms designed for static nodes [51].

\section{Based on Anchor nodes}

\subsection{Anchor based localization algorithm}

The nodes know their positions manually or by GPS. Target nodes localized by the known nodes are liable on the total of known nodes that affects accuracy.

\subsection{Anchor free localization algorithm}

The sensor nodes do not entail anchor nodes. Hence, the algorithm provides node positions that imply the location of the sensor nodes relative to each other [2].

\section{Based on GPS}

\subsection{Non-GPS based localization algorithm}

Without any additional hardware, the distance between the nodes computed. The localization begins from the known node and resorts to connectivity information to measure the hop count with the nodes in the network.

\subsection{GPS based localization algorithm}

The localization accuracy is too high; each node in the network needs a discrete GPS tracker. However, in a closed environment using GPS in all the nodes is unfeasible.

\section{Based on Region or Operating environment \\ 7.1 Indoor localization algorithm}

The localization of the sensor node inside the building will be more perplexing due to the deficient performance of GPS tracking. Where the environment is crowded and can have barriers like doors and walls, making the distance measurement as a tough task. Therefore,it is almost terrible to identify all nodes. The coverage of the wireless transmitter constrained due to signal attenuation. Wi-Fi hotspots, RSS fingerprint are capable solutions by its high accuracy and low infrastructure cost[18].

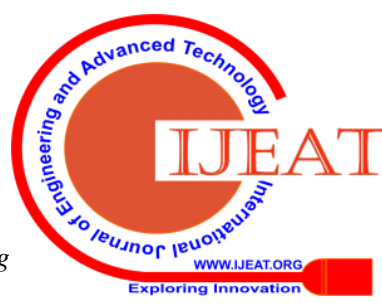




\subsection{Outdoor localization algorithm}

GPS is used to know the absolute position. However, accuracy tracking is not acceptable for the sports environment. In this kind of environment, the node to be estimated can be anywhere in the range.

\section{Based on communication}

\subsection{Non-Cooperative localization algorithm}

The location of each node established autonomously by using only source-anchor distance measurements [56].

\subsection{Cooperative localization algorithm}

Group of sensor nodes use distance measurement to estimate both position and angle of neighbor nodes, which trigger increased accuracy [37]. The notion in the rear is differential GPS, by which erroneous GPS measurements revised with range measurements from base stations using anchor nodes. It does not require additional hardware in some resource-constrained networks, and it results in costeffectiveness along with high-energy consumption [32].

\section{Based on Node density}

\subsection{Sparse localization algorithm}

For many ranging systems, the distance measurement is much less than that of communication ranges [2]. The accuracy for estimating the localization declines rapidly due to the lack of anchor nodes [47].

\subsection{Dense localization algorithm}

Accurate and efficient fingerprinting-built localization techniques depend on dense anchor nodes.

\section{Based on Coordinates \\ 10.1Absolute localization algorithm}

The sensor node needs additional hardware like GPS receiver to locate its position. The coordinates like latitude, longitude and altitude used. It obtained by a simple linear transformation and some reference nodes. Hence, it is easy to understand by a user [49].

\subsection{Relative localization algorithm}

Based on the application prerequisite, positions are either absolute or relative. Distance or angle defined by manual configuration or by some reference. This reduces overhead.

\section{COMPARATIVE STUDY}

In recent years, an extensive selection of localization algorithm for WSN introduced. Nasir et al [44] proposed Energy Harvesting Underwater Optical Wireless Sensor Networks (EH-UOWSNs) based on RSS localization structure, where seawater pose substantial encounters on the optical signal cause and network loss of range estimation. This reduces errors in estimating the shortest paths. Simulations reveal that the EH-UOWSNs, virtuous approach to get tough and precise results. Anjana et al. [7] presents a fault-resilient system for localization. This yields high precision with low communication overhead. The trial outcomes showed a direct connection amid the accuracy and range in localization. The Reference Point Group Mobility (RPGM) used to generate traces of mobile nodes by BonnMotion tool. Hence, Fault- Resilient Localization (FRL) is an energy efficient localization system for UWSNs.

Likewise, Nasir et al. [47] proposed a sturdy 3-D technique in localization for moderately linked UOWSNs, which can put up the outliers and enhance accuracy in the assignment of the known nodes. Xuan et al. [46] provides interesting solution for the problem in localization by developing the conditions under two sub-networks uniquely merged. The output displays that more than $90 \%$ of nodes in sparse 3-D networks localized. Yunfeng et al. [9] proposed a Range-Based Multilateral Accumulation Method (RBMAM), designed for large-scale, high-efficiency and high precision underwater networks. Here performance estimated along with LSM and ALS that prevails predominately and largely practiced in localization. Simulation evidences that accuracy in locating nodes and higher efficiency ideal for large-scale UWSN localization. Indoor localization [35] has been widely considered for the past decade. Among, RSS based localization of wireless techniques such as Wi-Fi or Bluetooth are widespread methods [36] due to its use in standard mobile devices, by providing relatively low infrastructure cost. To aim high localization accuracy by RSS fingerprint-based techniques, latest approaches have performed fusion between RSS-based localization and the user motion model [37].

Safa [3] proposed a novel method that exactly focuses in locating sensor by reducing power depletion and memory requests. Xiaoyong et al. [4] put forwarded a novel approach for multi-hop localization in WSN, based on nonlinear mapping and learning algorithm. Its performance analyzed, and the result shows high performance in location estimation adaptability. Fatemeh et al. [36] presents a meagre cost completely distributed in WSN localization technique, with appreciable accuracy. Experimental results unfold that this anticipated algorithm excels other range-free methods in homogeneous and heterogeneous WSNs. For performing with high precision of node localization in anisotropic WSNs with holes, Shi et al. [6] presented a Heuristic Multi-Dimensional Scaling (HMDS) algorithm. The Euclidean distance between nodes found by the virtual node and creating the shortest paths. This done by deploying the heuristic way through that it used to calculate accurate locations. This algorithm is precise and efficient compared to state-of-the-art methods in anisotropic WSNs. Saber et al. [5] proposed and executed a new process for geographic routing depending on a weighted centroid localization method, where the positions of target nodes are computed using fuzzy logic method. This method practices flow calculation over wireless channel to calculate the distance separating the anchor and sensor nodes. Huai et al. [8] put forward two localization methods. Here tri-directional coil affixed at an arbitrarily oriented position. They serve as both magnetic induction transmitter and receiver for wireless communications. This descends in approximating the distance of transmission and the polar angles based on the signals of to every known node. Kangshun et al. [10] presented a study for bringing up the precision in field of coal mines for the mobile node localization. Eke to nullify the influence affected by moving direction-offsets that hosted by the positioning systems. 
The ranging model obtained examined within the probabilistic framework. The suggested algorithm based on an overlapping self-adjustment approach and the anchor node selection. With low message transformation power consumption reduced. Pei et al. [18] put forward target node localization by data from both its one-hop and two-hop neighboring nodes. Thereafter Chebyshev center model coined to place a single target into its estimative region by a non-convex optimization problem overcome by Alternating Direction Method of Multipliers (ADMM) based PEPA. By presenting a hierarchical scheme, the Parallel Efficient Projection Algorithm (PEPA) extensively used to localize multiple targets with minimal communication cost. In addition to the above, a new heuristic error correction mechanism suggested enhancing the positioning precision. Amir et al. [17] present power saving on-demand protocol for the WSN-oriented indoor localization platform, the nodes sustained in a low energy idle listening mode by low energy built-in wake-up receiver. This starts only when a localization demand arises. This method allows motion time of the node that increases its battery life. Subrata et al. [45] presented method Minimization of Error in Multihop System (MEMHS). An ideal positioning approach led by placing maximum number of nodes in the network. The proposed algorithm has enhanced performance in error correction.

\section{RESULTS AND DISCUSSION}

We summarize the comparison outcomes against the performance parameter of localization algorithms in Table I. Here, we can infer that distributed algorithms are often more substantial than centralized algorithms. The centralized algorithms are proved by demonstration to be appreciable in the performance. However, distributed algorithms excel while sparsity of the network model was taken into consideration. In distributed method, computation cost and inter-node communication more than message transfer between base station and neighbor node. Likewise, range-free gives high accuracy. Similarly, paralleled to outdoor localization, indoor localization is more thought underneath a covering. Most of the algorithm influence due to mobility problem along various environmental discontinuities. The effect of sensor node density is not of much important for GPS free, as in GPS based.Table II shows the evaluation of existing localization techniques. The work presented in [5] - [6], [18], [37] describe range free algorithms. Generally, range free algorithms do toil very proficiently in unfriendly locations. Although precision may be high, scalability is low, and cost greater [5] [6]. Energy efficiency is missing both in [18][37], Localization error is low in [5][18][37]. Range based proposed in [10], [45], [48]. While node deployment is enticing with the prohibitive cost, these solutions are not acceptable, whereas energy efficient gives a promising future in the [45] and [48].

Devoid of low energy efficiency [9] is low cost, high accuracy, the localization error is low using MMSE method when the nodes deployed randomly. By its vanquishing nature hybrid category of localization [11] presents favorable low-cost factor, low localization error, high accuracy proposed an accurate mobile localization algorithm in a mine provoking due to the deficient performance of GPS

environment using LSM with uniform node deployment. An emerging category of localization proposed in [46] using a scalable error correction technique for the nodes deployed optimally over the network has proven energy-efficient and high accuracy. The results suggested in [4] and [8] offer a higher accuracy, lesser cost, and are very scalable. Looking into [10], the localization error alarms high that will not be a choice to remunerate localization in large area, though the accuracy is attractive and alluring despite low and energy efficiency is acceptable for underwater optical sensor networks [45]. The preceding solutions can only find position of minor part of nodes, but the algorithm proposed in [47] progress a new methodology to find conditions that two components distinctively combined in 3-D space. By this method, it meritoriously overwhelms error propagation in computing optimal component mergence parameters. HDMS algorithm used to find the Euclidean distances between nodes [7]. Using this algorithm communication complexity and computational complexity are applicable for the large-scale networks. Even though the rest of the caliber is conducive, yet cost factor is high for the hop-based category. It may be comparatively, and consent granted for the results tested in MATLAB.

Table -I: Performance parameter analysis of localization algorithm categories

\begin{tabular}{|l|l|l|l|l|l|l|l|l|l|}
\hline \multicolumn{1}{|c}{ Category } & $\begin{array}{c}\text { Localization } \\
\text { Technique }\end{array}$ & C & AC & S & EE & HS & SC & PC & TS \\
\hline Centralized & MDS, SDP & H & H & Y & H & L & N & H & R \\
\hline Distributed & & L & L & Y & L & H & Y & L & NR \\
\hline Range -based & RSSI & L & A & Y & H & L & Y & H & NR \\
\cline { 2 - 11 } & TDOA & L & H & Y & H & A & Y & H & R \\
\cline { 2 - 11 } & TOA & H & A & Y & L & H & Y & A & R \\
\cline { 2 - 11 } & AOA & H & L & N & N & H & Y & H & NR \\
\hline \multirow{5}{*}{ Range-free } & DV Hop & L & A & Y & H & L & N & L & R \\
\cline { 2 - 11 } & Hop Terrain & L & A & N & H & L & Y & L & R \\
\cline { 2 - 11 } & APIT & L & H & Y & H & A & Y & A & NR \\
\cline { 2 - 11 } & Centroid & L & H & Y & L & D & Y & H & NR \\
\cline { 2 - 12 } & Gradient & L & A & Y & H & A & Y & L & NR \\
\hline Fine -grained & & L & H & N & L & H & Y & L & R \\
\hline Coarse-grained & & L & L & Y & H & L & Y & H & NR \\
\hline Anchor based & & H & L & N & H & H & Y & H & R \\
\hline Anchor free & & L & A & Y & L & N & N & A & NR \\
\hline GPS based & & H & H & Y & H & H & N & H & R \\
\hline GPS Free & & L & A & N & L & L & Y & A & R \\
\hline
\end{tabular}

\begin{tabular}{lll} 
& \multicolumn{2}{c}{ Abbreviation } \\
AC-Accuracy & S-Security & EE-Energy efficiency \\
O-Optimum & A-Average & TS-Time synchronization \\
SC-Scalability & R-Required & PC-Power consumption \\
R-Random & D-Depends & ND-Node Deployment \\
C-Cost & H-High & LE- Localization error \\
Y-Yes & L-Low & NR-Not required \\
N-No & & HS-Hardware size
\end{tabular}


Table -II: Comparative analysis of recent localization algorithms

\begin{tabular}{|c|c|c|c|c|c|c|c|c|c|c|c|}
\hline $\begin{array}{l}\text { Year/ } \\
\text { Ref. }\end{array}$ & Category & Proposed System & $\begin{array}{c}\text { Technique/Method } \\
\text { Used }\end{array}$ & Parameters & ND & $\mathbf{E E}$ & AC & LE & $\mathbf{C}$ & Simulation & $\begin{array}{l}\text { Network } \\
\text { Area(m²) }\end{array}$ \\
\hline $\begin{array}{l}\text { Safa, } \\
\text { et al. } \\
2014[3]\end{array}$ & Distributed & $\begin{array}{l}\text { Hybrid DV-Hop } \\
\text { (HDV-Hop) } \\
\text { Algorithm }\end{array}$ & $\begin{array}{l}\text {-Linear Least } \\
\text { Square (LLS) } \\
\text {-Non-Linear Least } \\
\text { Square (NLLS) }\end{array}$ & $\begin{array}{l}\text {-Number of sensors } \\
\text {-Number of Anchors } \\
\text {-Area size }\end{array}$ & $\mathrm{D}$ & $\mathrm{Y}$ & $\mathrm{H}$ & $\mathrm{L}$ & $\mathrm{H}$ & $\begin{array}{l}\text { NS-2 } \\
\text { MATLAB }\end{array}$ & $\begin{array}{l}0.1 \mathrm{~km} \\
\text { to } \\
19 \mathrm{~km} 2\end{array}$ \\
\hline $\begin{array}{l}\text { Xiayong, } \\
\text { et al. } \\
\text { 2015[4] }\end{array}$ & Range-free & $\begin{array}{l}\text { Kernel Ridge } \\
\text { Regression - } \\
\text { Multi-hop } \\
\text { Localization } \\
\text { (KRR-ML) }\end{array}$ & $\begin{array}{l}\text {-Kernel based } \\
\text { regression } \\
\text { framework } \\
\text {-Learning method }\end{array}$ & $\begin{array}{l}\text {-Hop-count } \\
\text { information } \\
\text {-Position information }\end{array}$ & $\mathrm{R}$ & $\mathrm{Y}$ & $\mathrm{H}$ & $\mathrm{L}$ & $\mathrm{H}$ & MATLAB & $300 \times 300$ \\
\hline $\begin{array}{l}\text { Shizhang, } \\
\text { et al. } \\
\text { 2017[6] }\end{array}$ & $\begin{array}{c}\text { Range base/ } \\
\text { Range free }\end{array}$ & $\begin{array}{l}\text { Heuristic } \\
\text { Multi-Dimensional } \\
\text { Scaling (HMDS) } \\
\text { algorithm }\end{array}$ & Heuristic Approach & $\begin{array}{l}\text {-Euclidean distances } \\
\text {-Number of anchors } \\
\text {-Communication } \\
\text { range error }\end{array}$ & $\mathrm{R}$ & $\mathrm{N}$ & $\mathrm{L}$ & $\mathrm{L}$ & $\mathrm{L}$ & MATLAB & $100 \times 100$ \\
\hline $\begin{array}{l}\text { Saber, } \\
\text { et al. } \\
\text { 2017[5] }\end{array}$ & Range free & $\begin{array}{l}\text { Intelligent } \\
\text { mechanism for } \\
\text { routing Data based } \\
\text { on node } \\
\text { localization } \\
\text { (IMRL) }\end{array}$ & $\begin{array}{l}\text {-Weighted centroid } \\
\text { localization } \\
\text { technique } \\
\text {-Fuzzy logic } \\
\text { method }\end{array}$ & $\begin{array}{l}\text {-Energy consumption } \\
\text {-Packets } \\
\text {-Number of dead } \\
\text { nodes } \\
\text {-Transmission time }\end{array}$ & $\mathrm{R}$ & $\mathrm{Y}$ & $\mathrm{H}$ & A & $\mathrm{H}$ & MATLAB & $100 \times 100$ \\
\hline $\begin{array}{l}\text { Anjana, } \\
\text { et al. } \\
\text { 2017[7] }\end{array}$ & Mobile & $\begin{array}{l}\text { Fault-Resilient } \\
\text { Localization } \\
\text { scheme (FRL) for } \\
\text { UWSNs }\end{array}$ & $\begin{array}{l}\text { Multiple linear } \\
\text { regression } \\
\text { technique }\end{array}$ & $\begin{array}{l}\text {-Simulation time } \\
\text {-Communication } \\
\text { range } \\
\text {-Packet size }\end{array}$ & $\mathrm{D}$ & $\mathrm{Y}$ & $\mathrm{H}$ & $\mathrm{L}$ & $\mathrm{L}$ & $\begin{array}{l}\text { MATLAB } \\
\text { Aquasim }\end{array}$ & $\begin{array}{l}10 \mathrm{~km} \\
\text { to } \\
500 \mathrm{~km} \\
\end{array}$ \\
\hline $\begin{array}{l}\text { Huai, } \\
\text { et al. } \\
\text { 2018[8] }\end{array}$ & Sparse & $\begin{array}{l}\text { Rotation Matrix } \\
\text { (RM)-based } \\
\text { method } \\
\text { Distance-based } \\
\text { method }\end{array}$ & $\begin{array}{l}\text { Minimum } \\
\text { Mean-Square Error } \\
\text { (MMSE) }\end{array}$ & $\begin{array}{l}\text {-Space size } \\
\text {-Coil radius } \\
\text {-Excitation current }\end{array}$ & $\mathrm{R}$ & $\mathrm{N}$ & $\mathrm{H}$ & $\mathrm{L}$ & $\mathrm{L}$ & MATLAB & $20 \mathrm{~m}$ \\
\hline $\begin{array}{l}\text { Yunfeng, } \\
\text { et al. } \\
\text { 2018[9] }\end{array}$ & $\begin{array}{l}\text { Range } \\
\text { based }\end{array}$ & $\begin{array}{l}\text { Range-Based } \\
\text { Multilateral } \\
\text { Accumulation } \\
\text { Method (RBMAM) }\end{array}$ & $\begin{array}{l}\text {-Least Square } \\
\text { Method (LSM) } \\
\text {-Area Localization } \\
\text { Scheme (ALS) }\end{array}$ & $\begin{array}{l}\text {-Distance } \\
\text {-GPS data }\end{array}$ & $\mathrm{R}$ & $\mathrm{Y}$ & $\mathrm{H}$ & $\mathrm{H}$ & $\mathrm{H}$ & MATLAB & $\begin{array}{l}5000 x \\
5000\end{array}$ \\
\hline $\begin{array}{l}\text { Fatemeh, } \\
\text { et al. } \\
2018[36]\end{array}$ & $\begin{array}{l}\text { Range } \\
\text { free }\end{array}$ & $\begin{array}{l}\text { A distributed } \\
\text { cooperative and } \\
\text { range-free } \\
\text { localization } \\
\text { algorithm (DCRL) }\end{array}$ & Heuristic Method & $\begin{array}{l}\text {-Target neighbors } \\
\text {-Anchor nodes }\end{array}$ & $\mathrm{D}$ & $\mathrm{N}$ & $\mathrm{H}$ & $\mathrm{L}$ & $\mathrm{L}$ & MATLAB & $200 \times 200$ \\
\hline $\begin{array}{l}\text { Kangshun, } \\
\text { et al. } \\
2018[10]\end{array}$ & Hybrid & $\begin{array}{l}\text { An accurate mobile } \\
\text { localization } \\
\text { algorithm in a mine } \\
\text { environment }\end{array}$ & $\begin{array}{l}\text {-Error probability } \\
\text { approach } \\
\text {-Ooverlapping } \\
\text { self-adjustment } \\
\text { method } \\
\text {-LSM }\end{array}$ & $\begin{array}{l}\text {-Distance } \\
\text {-Time } \\
\text {-Distance Error }\end{array}$ & $\mathrm{U}$ & $\mathrm{Y}$ & $\mathrm{H}$ & $\mathrm{L}$ & $\mathrm{L}$ & MATLAB & $300 \times 8 \times 6$ \\
\hline $\begin{array}{l}\text { Subrata, } \\
\text { et al. } \\
\text { 2019[45] }\end{array}$ & $\begin{array}{l}\text { Hop } \\
\text { based }\end{array}$ & $\begin{array}{l}\text { Modified } \\
\text { Minimization of } \\
\text { Error in Multihop } \\
\text { System } \\
\text { M-MEMHS }\end{array}$ & $\begin{array}{l}\text { Scalable error } \\
\text { correction } \\
\text { technique }\end{array}$ & $\begin{array}{l}\text {-Number of nodes } \\
\text {-Transmission range }\end{array}$ & $\mathrm{O}$ & $\mathrm{Y}$ & $\mathrm{H}$ & $\mathrm{L}$ & $\mathrm{H}$ & MATLAB & $180 \times 180$ \\
\hline $\begin{array}{l}\text { Pei, } \\
\text { et al. } \\
\text { 2019[18] }\end{array}$ & $\begin{array}{l}\text { Range } \\
\text { free }\end{array}$ & $\begin{array}{l}\text { ADMM-based } \\
\text { parallel efficient } \\
\text { projection } \\
\text { algorithm (PEPA) }\end{array}$ & $\begin{array}{l}\text {-Chebyshev center } \\
\text { model } \\
\text {-Nonconvex } \\
\text { optimization } \\
\text { method }\end{array}$ & $\begin{array}{l}\text {-Number of Anchor } \\
\text { nodes } \\
\text {-Communication } \\
\text { range }\end{array}$ & $\mathrm{R}$ & $\mathrm{N}$ & $\mathrm{H}$ & $\mathrm{L}$ & $\mathrm{L}$ & MATLAB & $100 \times 100$ \\
\hline $\begin{array}{l}\text { Nasir, } \\
\text { et al. } \\
\text { 2019[44] }\end{array}$ & $\begin{array}{l}\text { Range } \\
\text { based }\end{array}$ & $\begin{array}{l}\text { Energy Harvesting } \\
\text { Underwater } \\
\text { Optical Wireless } \\
\text { Sensor Networks } \\
\text { (EH-UOWSNS) }\end{array}$ & $\begin{array}{l}\text { The minimum } \\
\text { unbiased variance } \\
\text { estimation method }\end{array}$ & $\begin{array}{l}\text {-Number of Anchor } \\
\text { nodes } \\
\text {-Coordinates }\end{array}$ & $\mathrm{R}$ & $\mathrm{N}$ & $\mathrm{H}$ & $\mathrm{L}$ & $\mathrm{H}$ & MATLAB & $100 \times 100$ \\
\hline $\begin{array}{l}\text { Xuanlin, } \\
\text { et al. } \\
\text { 2019[46] }\end{array}$ & $\begin{array}{l}\text { Range } \\
\text { based }\end{array}$ & $\begin{array}{l}\text { Component-based } \\
\text { localization (CBL) }\end{array}$ & $\begin{array}{l}\text {-Algebra-based } \\
\text { approach } \\
\text {-Patch-and-stitching } \\
\text { strategy }\end{array}$ & $\begin{array}{l}\text {-Node degree } \\
\text {-Anchor ratio } \\
\text {-Distance } \\
\text { measurement error }\end{array}$ & $\mathrm{D}$ & $\mathrm{Y}$ & $\mathrm{H}$ & $\mathrm{L}$ & $\mathrm{H}$ & MATLAB & $\begin{array}{l}\text { 10Rx10R } \\
\text { x10R }\end{array}$ \\
\hline $\begin{array}{l}\text { Nasir, } \\
\text { et al. } \\
2019 \text { [47] }\end{array}$ & $\begin{array}{c}\text { 3D } \\
\text { Deployment }\end{array}$ & $\begin{array}{l}\text { Robust 3D } \\
\text { localization for } \\
\text { UOWSNS }\end{array}$ & $\begin{array}{l}\text { Low-rank matrix } \\
\text { approximation } \\
\text { method }\end{array}$ & $\begin{array}{l}\text {-Number of Anchor } \\
\text { nodes } \\
\text {-Coordinates }\end{array}$ & $\mathrm{R}$ & $\mathrm{N}$ & $\mathrm{H}$ & $\mathrm{L}$ & $\mathrm{H}$ & \begin{tabular}{c|}
$3 \mathrm{D}$ \\
environment
\end{tabular} & $\begin{array}{l}10 x 10 x \\
10\end{array}$ \\
\hline
\end{tabular}




\section{CONCLUSION}

In this paper, we explore and expedite the choice of prevailing adequate localization algorithms with various parameters and techniques. By combining data with various physical values from different localization narrowed down to the progress of many kinds of open research challenge. This will enhance in acclimatizing the localization errors and achieve high precision in large-scale centralized network localization to decrease the computational complexity. The performance issues had to venture into several tough tasks quoted by the above-cited WSN features on the one hand and the demands of the applications on the other. The latest research show that mobility in WSNs improves the volume of data in the network, addressing delay and latency glitches.

In future work, to promote the positioning of node on vast region and to improve performance, usage of intelligent techniques is optimal. Likewise, discovery of efficient energy routing protocol intended for robustness, and to find accuracy in peer-assisted localization there is a desperate need for the still tuned potent technologies for specific need and application like Internet of Things, UWSN domain and human-machine cooperation

\section{REFERENCES}

1. Tashnim J.S. Chowdhury, Colin Elkin, Vijay Devabhaktuni, Danda B. Rawat, Jared Oluoch, "Advances on localization techniques for Wireless Sensor Networks: A survey," Elsevier Journal on Computer Networks, Volume 110, 2016, Pages 284-305.

2. Nikhil Deshpande, Edward Grant and Thomas, C. Henderson, "Target Localization and Autonomous Navigation Using Wireless Sensor Networks-A Pseudo gradient Algorithm Approach," IEEE journal on systems, Volume 8, Issue 1,2014, Pages 93-103.

3. Haidar Safa, "A novel localization algorithm for large scale Wireless Sensor Networks," Elsevier Journal on Computer Communications, Volume 45,2014, Pages 32-46.

4. Xiaoyong Yan, Aiguo Song, Zhong Yang, Wankou Yang,“An improved multihop-based localization algorithm for wireless sensor network using

5. learning approach," Elsevier Journal on Computers and Electrical Engineering, Volume 48, 2015, Pages 247-257.

6. Saber Amri, Fekher Khelifi, Abbas Bradai, Abderrezak Rachedi, Med Lassaad Kaddachi, Mohamed Atri, "A new fuzzy logic-based node localization mechanism for Wireless Sensor Networks",Elsevier Journal on Future Generation Computer Systems, 2017.

7. Shi Zhang, MengJooEr, Baihai Zhang, Yashar Naderahmadian, "A novel heuristic algorithm for node localization in anisotropic wireless sensor networks with holes," Elsevier Journal on Signal Processing, Volume 138,2017, Pages 27-34.

8. Anjana P Das,Sabu M Thampi, "Fault-resilient localization for underwater sensor networks", Elsevier Journal on Ad-Hoc Networks, Volume 55,2017, Pages 132-142.

9. HuaiHuang, Yahong Rosa Zheng "3-D localization of wireless sensor nodes using near- field magnetic induction communications", Elsevier Journal on Physical Communication, Volume 30 ,2018, Pages97-106.

10. Yunfeng Han, Dajun Sun, Cuie Zheng1, Jucheng Zhang, "Centralized underwater node localization using Range Based
Multilateral Accumulation Method (RBMAM)", Elsevier Journal on Applied Acoustics, Volume138, 2018, Pages 115-120.

11. Kangshun Li, Hui Wang, Shanni Li, "A mobile node localization algorithm based on an overlapping self-adjustment mechanism," Elsevier Journal on Information Sciences, Volume 481,2018, Pages 635-649.

12. L. Doherty,K.S.J.pister, L. El Ghaoui, "Convex position estimation in Wireless Sensor Networks", IEEE Journal on Computer and Communications Societies, Volume 3, 2001, Pages 1655-1663.

13. X. Shi, G. Mao, B.D. Anderson, Z. Yang, J. Chen, "Robust localization using range measurements with unknown and bounded errors," IEEE Transaction on Wireless Communication, Volume16, Issue 6, 2017, pages 4065-4078.

14. Z. Wang, H. Zhang, T. Lu, T.A. Gulliver, "A grid-based localization algorithm for Wireless Sensor Networks using connectivity and RSS rank," IEEE Journal, Volume 6, 2018, Pages 8426-8439.

15. J.-P. Sheu, P.-C. Chen, C.-S. Hsu, "A distributed localization scheme for wireless sensor networks with improved grid-scan and vector-based refinement," IEEE Transaction on Mobile Computing, Volume7, Issue9,2008, Pages 1110-1123.

16. Q. Shi, C. He, H. Chen, L. Jiang, "Distributed wireless sensor network localization via sequential greedy optimization algorithm,” IEEE Transaction on Signal Process, Volume 58 (6),2010, Pages 3328-3340.

17. F. Shahzad, T.R. Sheltami, E.M. Shakshuki, "Dv-Max hop: a fast and accurate range-free localization algorithm for anisotropic wireless networks," IEEE Transaction on Mobile Computing, Volume16, Issue 9 ,2017, Pages 2494-2505.

18. Amir Guidara, Faouzi Derbel, Ghofrane Fersi, Sadok Bdiri, Maher Ben Jemaa, "Energy-efficient on-demand indoor localization platform based on Wireless Sensor Networks using low power wake up receiver," Elsevier Journal on Ad Hoc Networks, Volume 93 ,2019, Page 101.

19. Pei Xie, Keyou You,Shiji Song, Cheng Wu, "Distributed range-free localization via hierarchical nonconvex constrained optimization", Elsevier Journal on Signal Processing, Volume 164,2019, Pages 136-145.

20. Aditya Vempaty, Yunghsiang S. Han, Pramod K.Varshney, "Target Localization in Wireless Sensor Networks Using Error Correcting Codes", IEEE Transactions on Information Theory, Volume 60, Issue: 1, January 2014, Pages 697-712.

21. NoureddineLasla, Mohamed F. Younis, Abdelraouf Ouadjaout, "An effective Area-Based localization algorithm for wireless networks," IEEE transactions on computers, Volume 64, issue 8, 2015.

22. Linqing Gui, Thierry Val, Anne Wei, Réjane Dalce, "Improvement of range-free localization technology by a novel DV-hop protocol in Wireless Sensor Networks," Elsevier Journal on Ad-Hoc networks, Volume 24,2015, Pages 55-73.

23. Aspnes, J., T. Eren, D.K. Goldenberg, A.S. Morse, W. Whiteley, Y.R. Yang, B.D. Anderson, and P.N.Belhumeur, "A theory of network localization", IEEE Transactions on Mobile Computing,2006, Pages 1663-1678. 
24. Guoqiang Mao, BarışFidan, Brian D.O.Anderson, "Wireless sensor network localization techniques", Elsevier Journal on Computer and Telecommunications Networking, Volume 51, Issue 10,2007, Pages 2529-2553.

25. Jennifer Yick, Biswanath Mukherjee, Dipak Ghosal "Wireless sensor network survey," Elsevier Journal on Computer Networks, Volume 52, 2008, Pages 2292-2330.

26. Koen Langendoen, Niels Reijers, "Distributed localization in wireless sensor networks: a quantitative comparison," Elsevier Journal on Computer and Telecommunications Networking, Volume 43, Issue 4, 2003.

27. Lazos, Loukas, and Radha Poovendran, "HiRLoc: High-Resolution robust Localization for Wireless Sensor Networks," Selected Areas in Communications, IEEE Journal on 24, Issue 2, 2006, Pages 233-246.

28. C. Luo, W. Li, H. Yang, M. Fan, X. Yang, "Mobile target positioning using refining distance measurements with inaccurate anchor nodes in chain-type Wireless Sensor Networks", Springer Journal on Mobile Networks \& Applications, Volume 19, Issue 3,,2014,Pages363-381.

29. G.A. Shah, O.B. Akan, "Timing-based mobile sensor localization in wireless sensor and actor networks," Springer Journal on Mobile Network Application, Volume15, Issue5,2010, Pages 664-679.

30. E. Xu, Z. Ding, S. Dasgupta, "Target tracking and mobile sensor navigation in Wireless Sensor Networks," IEEE Transaction on Mobile Computing, Volume12, Issue1,2013, Pages177-186.

31. H. Chen, F. Gao, M. Martins, P. Huang, J. Liang, "Accurate and efficient node localization for mobile sensor networks," Elsevier Journal on Mobile Network Application, Volume18, Issue1,2013, Pages141-147.

32. W. J. Feng, X.W. Bi, R. Jiang," A novel adaptive cooperative location algorithm for Wireless Sensor Networks," International Journal on Automation Computing, volume 9, issue 5,2012, Pages 539-544.

33. Y. Ahmadi, N. Neda and R. Ghazizadeh, "Range-Free Localization in Wireless Sensor Networks for Homogeneous and Non-Homogeneous Environment," in IEEE Journal on Sensors, volume. 16, issue 22,2016, Pages 8018-8026.

34. S. He, S.-H. Chan, "Wi-Fi Fingerprint-based indoor positioning: Recent advances and comparisons," IEEE Communication Survey Tutorial, Volume18, Issue1,2016, Pages466-490.

35. R. Faragher, R. Harle, "Location fingerprinting with Bluetooth low energy beacons," IEEE Journal of Selected areas communication, volume33, issue 11, 2015, Pages2418-2428.

36. Z. Yang, C. Wu, Z. Zhou, X. Zhang, X. Wang, Y. Liu, "Mobility increases localizability: A survey on wireless indoor localization using inertial sensors," ACM Computation Survey, volume 47, issue3,2015, Pages 54.

37. Fatemeh Darakeh, Gholam-Reza Mohammad-Khani, PaeizAzmi, "DCRL-WSN: A distributed cooperative and range-free localization algorithm for Wireless Sensor Networks," Elsevier International Journal on Electronic Communication, Volume 93 ,2018, Pages 289-295.

38. Joe-Air Jiang, Xiang-Yao Zheng, Yu-FanChen, Chien-Hao Wang, Po-Tang Chen, Cheng-Long Chuang, Chia-Pang Chen, "A distributed RSS-Based Localization Using a Dynamic Circle Expanding Mechanism,” IEEE Sensors Journal, Volume 13, Issue 10, 2013, Pages $3754-3766$.
39. Chien-Yih Wan, L.Krishnamurthy, "Pump-Slowly, Fetch-Quickly (PSFQ): A Reliable Transport Protocol for Sensor Networks", IEEE Journal on selected areas in Communications, Volume 23, issue 4, 2005.

40. Hojae Lee, Sanghoon Lee and Yeonsoo Kim, Hakjin Chong, "Grouping Multi-Duolateration Localization using Partial Space Information for

41. Indoor Wireless Sensor Networks," IEEE Transaction on Consumer Electronics, Volume55, issue 4, 2009, Pages1950 1958.

42. EdoardoAmaldi, Antonio Capone, Matteo CesanaIlario Filippini, "Design of Wireless Sensor Networks for Mobile Target Detection," IEEE Journal on Networking, Volume 20, Issue 3, 2012, Pages384-397.

43. Shancang Li, Xinheng Wang, Shanshan Zhao, Jue Wang, Ling Li, "Local Semi-definite Programming-Based Node Localization System for Wireless Sensor Network Applications," IEEE Systems Journal, Volume 8, 2014, Pages879 - 888.

44. B. Liu, H. Chen, Z. Zhong, and H. V. Poor, "Asymmetrical round-trip based synchronization-free localization in large-scale underwater sensor networks," IEEE Transactions on Wireless Communication, Volume 9, Issue 11,2010, Pages 3532-3542.

45. A. Y. Teymorian, W. Cheng, L. Ma, X. Cheng, X. Lu, and Z. $\mathrm{Lu}$, "3D underwater sensor network localization", IEEE transactions on Mobile Computation, Volume 8, Issue 12,2009, Pages1610-1621.

46. Nasir Saeed, Tareq Y. Al-Naffouri, Abdulkadir Celik, Mohamed-Slim Alouini, "Localization of Energy Harvesting Empowered Underwater Optical Wireless Sensor Networks," IEEE transactions on wireless communications, Volume18, Issue 5, 2019, Pages 2652-2664.

47. Subrata Dutta, Keshav Dahal, Mohammad S. Obaidat, Debasis Giri, Sarmistha Neogy, "M-MEMHS: Modified Minimization of Error in Multihop System for Localization of unknown Sensor Nodes," IEEE systems journal, Volume13, Issue 1, 2019, Pages 215-226.

48. Xuan Liu, Jiangjin Yin, Shigeng Zhang, Bo Ding, Song Guo, Kun Wang "Range-Based Localization for Sparse3-D Sensor Networks", IEEE Journal on Internet of Things, Volume 6, Issue 1, 2019, Pages 753-765.

49. Nasir Saeed, Tareq Y. Al-Naffouri, Mohamed-Slim Alouini, "Outlier Detection and Optimal Anchor Placement for 3-D Underwater Optical Wireless Sensor Network Localization", IEEE transactions on communications, Volume 67, Issue 1, 2019, Pages 611-623.

50. Guangjie Han, Huihui Xu,Trung Q. Duong, Jinfang Jiang, Takahiro Hara, "Localization algorithms of Wireless Sensor Networks: a survey", Springer Journal of Telecommunication Systems, 2013, Volume 52, Issue 4, Pages 2419-2436.

51. Wang, J., Ghosh, R.K. \& Das, S.K., “A survey on sensor localization" , Springer Journal of Control Theory and Applications, 2010, Volume 8, Issue 1, Pages 2-11.

52. Suprakash Datta, Chris Klinowski, Masoomeh Rudafshani and Shaker Khaleque, "Distributed localization in static and mobile sensor networks," IEEE International Conference on Wireless and Mobile Computing, Networking and Communications, 2006, Pages: 69 - 76 
53. Jianying Zheng; Yan Huang," A range-free localization algorithm based on the velocity information for mobile sensors," IEEE International Conference on Intelligent Computing and Intelligent Systems, 2010, Volume: 1, Pages: $589-593$

54. Jang-Ping Sheu; Wei-Kai Hu; Jen-Chiao Lin, "Distributed Localization Scheme for Mobile Sensor Networks," IEEE Transactions on Mobile Computing, 2010, Volume 9, Issue 4, Pages 516 - 526.

55. A. E. Assaf, S. Zaidi, S. Affes, and N. Kandil, "Low-cost localization for multihop heterogeneous Wireless Sensor Networks," IEEE Transaction Wireless Communication, Volume 15, Issue 1, Pages 472-484, 2016.

56. S. Srirangarajan, A. H. Tewfik, and Z.-Q. Luo, "Distributed sensor network localization using SOCP relaxation," IEEE Transaction Wireless Communication, Volume 7, Issue 12, Pages 4886-4895, 2008.

57. S. Tomic, M. Beko, and R. Dinis, "RSS-based localization in wireless sensor networks using convex relaxation: Noncooperative and cooperative schemes," IEEE Transaction Vehicular Technology, Volume 64, Issue 5, Pages 2037-2050, 2015.

\section{AUTHORS PROFILE}

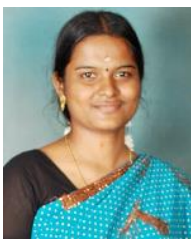

Panimalar. K. received her B.Tech 2009 in and M.Tech 2011 in Computer Science and Engineering from Pondicherry University. Currently doing research in Department of Computer Science and Engineering, Pondicherry Engineering College, Puducherry. She is Member of ISTE, India.

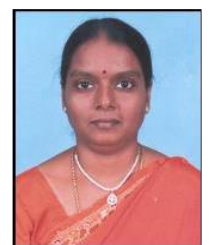

Dr. S. Kanmani received her B.E and M.E in Computer Science and Engineering from Bharathiyar University and Ph.D. from Anna University, Chennai. She had been the faculty of Department of Computer Science and Engineering, Pondicherry Engineering College from 1992 onwards. Presently she is Professor in Department of Information Technology, Pondicherry Engineering College, Puducherry. Her research interests are Software Engineering, Software Testing, Object-Oriented System, and Data Mining. She is Member of Computer Society of India, ISTE and Institute of Engineers, India. She has published about 50 papers in various International conferences and journals. 